\title{
Intra-Stroke Profiling of Wheelchair Propulsion Using Inertial Measurement Units ${ }^{\dagger}$
}

\author{
Amy R. Lewis ${ }^{1,2, *}$, Elissa J. Phillips ${ }^{2}$, William S. P. Robertson ${ }^{1}$, Paul N. Grimshaw ${ }^{1}$ and \\ Marc Portus ${ }^{2}$ \\ 1 School of Mechanical Engineering, University of Adelaide, Adelaide 5005, Australia; \\ will.robertson@adelaide.edu.au (W.S.P.R.); paul.grimshaw@adelaide.edu.au (P.N. G.) \\ 2 Movement Science, Australian Institute of Sport, Canberra 2617, Australia; \\ elissa.phillips@ausport.gov.au (E.J.P.); marc.portus@ausport.gov.au (M.P.) \\ * Correspondence: amy.lewis@adelaide.edu.au; Tel.: +61-8-8313-4805 \\ + Presented at the 12th Conference of the International Sports Engineering Association, Brisbane, \\ Queensland, Australia, 26-29 March 2018.
}

Published: 23 February 2018

\begin{abstract}
The instrumentation of wheelchair racing chairs is currently limited by the influence of the athlete-wheelchair system mass and performance. Inertial measurement units (IMUs) provide a lightweight solution, and have demonstrated accurate kinematic monitoring of wheelchair ball sports. With the aim of investigating the capability of IMUs detecting contact and release timings, a study was performed on seven national level wheelchair racing athletes (T34 and T54 classifications). Athletes performed treadmill-based propulsion at speeds ranging between $19 \mathrm{~km} / \mathrm{h}$ and $32 \mathrm{~km} / \mathrm{h}$, with the population sample enabling comparison between classification, propulsion speed, gender, age, glove type and wheel type. Contact and release timing points of each athlete were verified against measures obtained using video capture $(100 \mathrm{~Hz})$, which is considered a gold standard measure. IMUs identified contact timings for all athletes, however could not consistently identify release points for all athletes. Propulsion speed and gender were found to have minimal influence on the capacity to determine contact.
\end{abstract}

Keywords: wheelchair propulsion; instrumentation; inertial measurement units

\section{Introduction}

With the winning margins of wheelchair racing events being within hundredths of a second, the use of an optimal propulsion technique, and its quantitative assessment on-track is critical to an athlete's success. Stroke speed, stroke length (time between successive contacts), push times (time when the hand is in contact with pushrim) and recovery times represent key characteristics important for the kinematic analysis of wheelchair propulsion. These parameters are a crucial requirement in diagnosing the efficiency of the interaction of force transfer between the hand and wheel. Additionally, it is crucial to understand the relative timings between the hands (kinematic asymmetries), which are common across the athletic and non-athletic wheelchair populations, and are often a result of the type of physical impairment [1]. Asymmetries can negatively impact propulsion, such as through steering difficulty $[2,3]$ and so kinematic measurement of asymmetry requires the accurate identification of key propulsion parameters including contact times [4].

Inertial measurement units (IMUs) have demonstrated success in the evaluation of wheelchair kinematics [5]. Previous research has been constrained to the determination of wheelchair contact and hence stroke time, however has not been extended into intra-stroke profiling. Use of IMUs can provide in-field assessment to measure the variability in propulsion during a race, which is otherwise unavailable using the current gold standard of motion capture (kinematics) or use of instrumented 
wheels (kinetics). Although instrumented wheels such as the SMARTwheel are commercially available, they are infrequently implemented by wheelchair racing athletes, as the wheel mass is of comparable magnitude to the wheelchair itself [6,7], with increases in system mass demonstrated as being detrimental to performance [8]. Additionally, use of IMUs can provide an objective method of identifying contact, which is less subject to parallax and perspective errors of video analysis. Following the development of code to automate acceleration data to accurately and reliably identify contact, data processing becomes a semi-automated process, with substantially reduced computational requirements as compared with the manual digitisation of video, the current gold standard measurement method [5].

The ability of the IMU to identify propulsion characteristics is reliant on the underlying noise of the signal, as well as athlete equipment (such as glove and wheel types), and technique, which influences the magnitude of peak impacts. Wheelchair racing athletes typically wear gloves as a form of protection from the ballistic striking motion encountered in wheelchair racing. These gloves are typically made from leather or a thermoplastic. The variation in the stiffness of these gloves, or the use of disc and spoked wheels can influence the characteristic acceleration signal demonstrating contact between the athlete and wheelchair.

The aim of this research was to determine the capability of IMUs in automatically identifying both contact and release times (and hence push time) across a number of different athlete and wheelchair configurations as a measure of robustness of the solution.

\section{Materials and Methods}

\subsection{Athlete Selection}

Seven wheelchair racing athletes from the national level junior $(n=4,<19$ years of age, $4.0 \pm 0.58$ years of experience) and senior ( $n=3,>19$ years of age, $8.33 \pm 3.28$ years of experience) program were recruited for this study (Table 1). The population sample facilitated assessment of the robustness of the method across varying athlete anthropometries (mass and gender), push technique (propulsion speed), athlete ability (classification and age (junior or senior)), and equipment (wheel type and glove type). Research was constrained to classifications demonstrating fair to good functional strength in the arms and trunk (T53, T54: Spinal Cord Injury; T34: Cerebral Palsy) [9]. Further classifications and wheelchair sports were excluded to prevent demographics confounding observations (i.e., seating posture, physical impairment in upper extremity). Athletes provided written, informed consent, with ethical approval obtained by the University of Adelaide and the Australian Institute of Sport.

Table 1. Athlete demographics. System mass represents both athlete and wheelchair mass.

\begin{tabular}{cccccccc}
\hline Athlete & $\begin{array}{c}\text { System } \\
\text { Mass } \mathbf{( k g )}\end{array}$ & Classification & $\begin{array}{c}\text { Propulsion Speed } \\
\mathbf{( k m} / \mathbf{h})\end{array}$ & Gender & Age & $\begin{array}{c}\text { Glove } \\
\text { Type }\end{array}$ & $\begin{array}{c}\text { Wheel } \\
\text { Type }\end{array}$ \\
\hline A1 & 60.14 & T34 & 21,22 & $\mathrm{M}$ & Junior & Soft & Disc \\
A2 & 62.50 & $\mathrm{~T} 34$ & 19,20 & $\mathrm{M}$ & Junior & Soft & Disc \\
A3 & 60.32 & $\mathrm{~T} 54$ & $22,23,24$ & $\mathrm{~F}$ & Senior & Hard & Disc \\
A4 & 54.43 & $\mathrm{~T} 54$ & $26,27,28$ & $\mathrm{~F}$ & Senior & Hard & Disc \\
A5 & 62.20 & $\mathrm{~T} 54$ & $29,30,32$ & $\mathrm{M}$ & Senior & Hard & Disc \\
A6 & 87.93 & $\mathrm{~T} 54$ & 26,27 & $\mathrm{M}$ & Junior & Soft & Spoke \\
A7 & 54.31 & $\mathrm{~T} 54$ & 22,23 & $\mathrm{M}$ & Junior & Hard & Spoke \\
\hline
\end{tabular}

\subsection{Experimental Propulsion Task}

Athletes completed trials at constant velocity on a treadmill $\left(\mathrm{H} / \mathrm{P} / \mathrm{Cosmos}^{\circledR}\right.$ Saturn, Traunstein, Germany) inclined to $1 \%$ [10]. Belt velocity was comparable to individual regular race training velocity $(92.12 \% \pm 2.36 \%$ of mean race velocity at an international competition held within six months of data collection [11,12]). Athletes who were new to treadmill propulsion completed a familiarisation protocol, whereby treadmill velocity was incrementally increased until testing velocity was reached. A fiveminute active recovery period was provided between trials to minimise effects of fatigue. 
The front fork of the wheelchair was fastened to the treadmill using a custom designed clamp, which prevented lateral translations of the wheelchair, but allowed normal fore-aft movement of the wheelchair within a safe operating range (Figure 1a). Any potential detriments to athlete performance from treadmill based propulsion did not impede the capabilities of the IMU in measuring the temporal characteristics of propulsion.

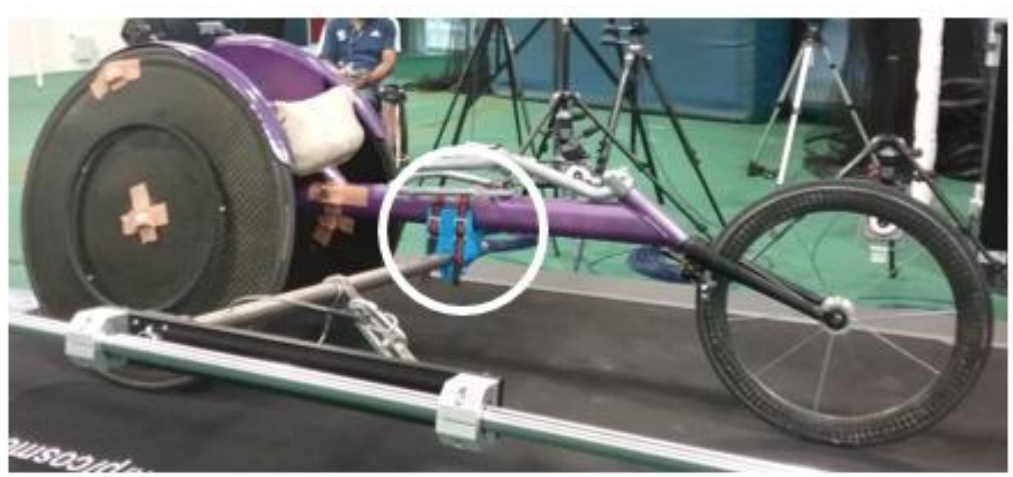

(a)

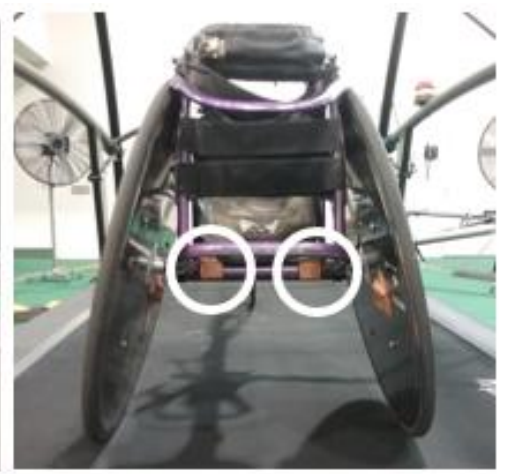

(b)

Figure 1. Testing set up. (a) Custom clamp (circled) keeping wheelchair on treadmill; (b) IMU locations (circled).

\subsection{Measurement System}

Triaxial acceleration data were collected using two synchronised IMUs (Figure 1b; IMeasureU, Auckland, New Zealand; Accelerometer: \pm 16 g; Gyroscope: $\pm 2000 \%$; Compass: $\pm 1200 \mu \mathrm{T}$ ) sampling at $500 \mathrm{~Hz}$. Units were located on the left and right axle housings of the wheelchair frame (FL, FR). Each unit was carefully fasted using double sided adhesive tape and secured using fabric tape to prevent all unwanted lateral translations and vibrations of the units. Pilot testing revealed the accelerations measured by the IMU were low (contact peak $<4 \mathrm{~g}$ ) with respect to the sensing range, suggesting the IMUs used were appropriate for this research.

Three high speed Sony PXW-FS7 video cameras (located at the rear and perpendicularly left and right of the athlete-treadmill system) were utilised to validate the push durations calculated by the IMUs. Cameras operated at a sampling frequency of $100 \mathrm{~Hz}$ and shutter speed of $1 / 1000 \mathrm{~s}$. Three distinct contacts were made between the hands and wheels followed by a stationary period of $20 \mathrm{~s}$ to synchronise IMU and video data.

\subsection{Data Processing}

Resultant accelerometer data were all low pass filtered with a bidirectional low-pass filter using a $-6 \mathrm{~dB}$ cut off frequency of $100 \mathrm{~Hz}$, which was determined through performing independent residual analyses for each IMU, athlete and speed. $100 \mathrm{~Hz}$ represented the highest recommended cut off frequency, and was nominated due to the low likelihood of altering temporal characteristics. The resultant acceleration vector was used. This accounted for orientation discrepancies during placement and ensured data for both FL and FR are in the same global coordinate system.

Contact and release points were automatically detected using a custom peak-detection algorithm which located local maxima, with push time calculated as the time between successive contacts. The algorithm specified minimum distance between like points (contact or release) was $0.45 \mathrm{~s}$ based on previous literature [13], with athlete-specific minimum peak thresholds manually defined based on visual inspection $\left(0.3-17 \mathrm{~m} / \mathrm{s}^{2}\right)$. Temporal parameters were validated against video, which served as the gold standard measurement [5]. Video data was manually digitised (Kinovea v0.8.15 www.kinovea.org) with hand contact and release identified as the first and last frame where any part of the athlete's hand or glove was in contact with the pushrim, respectively. 


\subsection{Statistical Analysis}

Reliability and repeatability measures were obtained through Intraclass Correlation Coefficients (ICC). Two independent raters (both familiar with wheelchair acceleration data and video) performed manual digitisation on the same video, and analysed IMU data using the custom peak detection algorithm. Both raters performed a repeated assessment of tasks following a period of two weeks with no exposure to the data. Appropriate reliability and repeatability required ICC $>0.9$ [14].

A mathematical relationship established the agreement between the video-based and IMUbased contact timing data. It was defined for this research that an adjusted coefficient of determination $\left(R^{2}\right)$ value $>0.97\left( \pm 1\right.$ video frame $(0.01 \mathrm{~s})$ ) demonstrated excellent goodness of fit, $R^{2}>$ $0.91( \pm 2$ video frames $(0.02 \mathrm{~s}))$ was very good, $R^{2}>0.80( \pm 3$ video frames $(0.03 \mathrm{~s}))$ was good, with $R^{2}<$ 0.80 considered average. Results of the above regression were then ranked and compared across athlete demographics using a Mann Whitney U test. Pearson Correlations were used to establish the relationship present between speed and reliability. All statistical calculations were performed using IBM SPSS Statistics 21 Software for Windows (SPSS Inc., Chicago, IL, USA).

A total of 221 push times were analysed ( 4 junior athletes $\times 13$ push times $\times 2$ speeds +3 senior athletes $\times 13$ push times $\times 3$ speeds) for each hand (442 total).

\section{Results}

Excellent inter- and intra-rater reliability (ICC $>0.95$ ) were obtained for the manual digitisation of high speed video. For all reliability assessments, mean absolute error in the time for push length was $<0.013 \mathrm{~s}$, with standard deviation being $<0.058 \mathrm{~s}$ and $<0.013 \mathrm{~s}$ for IMU, and video defined contacts, respectively.

IMUs were effectively capable of automatically identifying contact, however the algorithm located release less reliably, without also detecting noise (Figure 2). At this stage automatic detection of release points is not plausible without the assistance of VICON data. As the release point was not always reliably detected, the remainder of this results section will focus solely on the reliability of identifying contact.

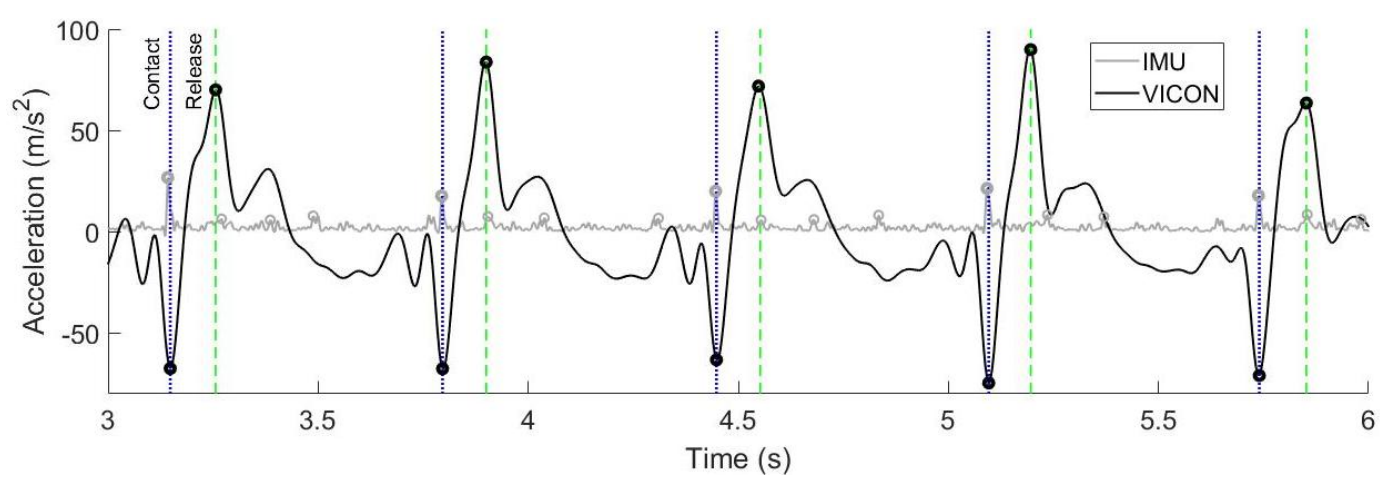

Figure 2. Representative example of acceleration trace demonstrating the clear detection of contact points with an IMU, however issues with the identification of release.

Athlete-specific characteristics were observed to influence the robustness of the IMU method, and hence the level of agreement with the video obtained contact timings. Results from the Mann $U$ Whitney test revealed that, glove type $(\mathrm{U}=6 ; \mathrm{W}=34 ; p=0.02)$, and wheel type $(\mathrm{U}=12 ; \mathrm{W}=22 ; p=$ 0.26) were not observed to impede the effectiveness of IMUs in determining contact (Figure 3 ). Statistical affects were identified between the reliability of IMU and video derived push lengths, with speed $(r=0.72 ; p<0.01)$, gender $U=0 ; W=55 ; p=0.05)$, athlete classification $(U=4 ; W=14 ; p=0.02)$ and age $(\mathrm{U}=0 ; \mathrm{W}=36 ; p<0.01)$, or a compounding affect between these. Effect sizes ranged between small (Wheel $r=0.05$; Gender $r=0.05$ ), medium (Glove $r=0.11$; Age $r=0.15$; Classification $r=0.11$ ) and large (Speed $r=0.72)$ [15]. 

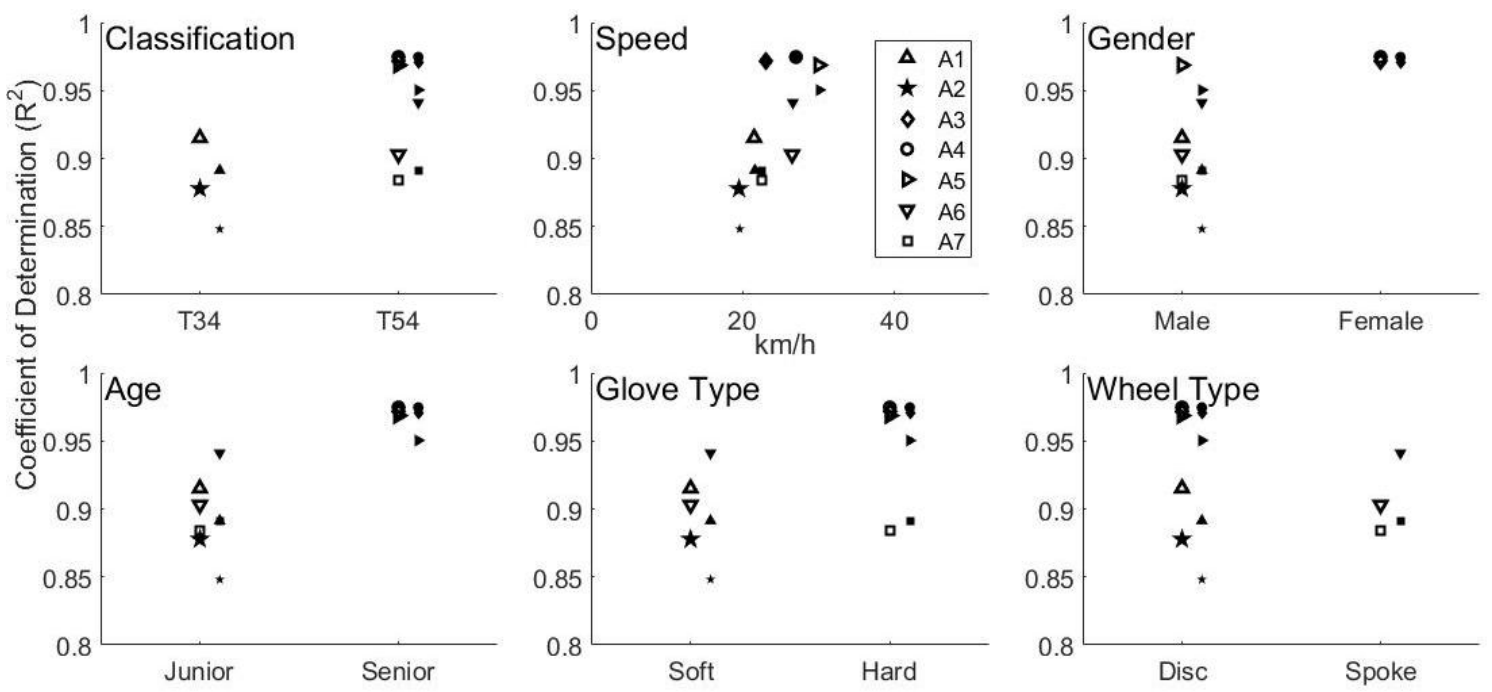

Figure 3. Capability assessment of the accuracy of the IMU based contact detection methodology across varying athlete characteristics as presented in Table 1. Coefficient of determination $\left(R^{2}\right)$ data are presented from the FL (open markers) and FR (solid markers) for each athlete.

\section{Discussion}

The aim of this research was to investigate the ability of IMUs to perform intra-stroke profiling of wheelchair propulsion, and the influence of athlete and wheelchair demographics on this ability. It was observed that IMUs provided a reliable and repeatable method of identifying contact, but not the release point. Release was able to be detected, however as acceleration signals at this moment were comparable to the magnitude of signal noise, it may not allow completely autonomous processing. The accuracy of the IMU method was influenced both through placement location and athlete technique. Due to the high reliability of the IMU methodology, it is plausible to perform automated, in-field analysis of propulsion, and analyse the presence of asymmetry during motion.

Contact is a far more ballistic motion than release of the hand on the pushrim, which can explain the reduced accuracy of its detection. A single athlete demonstrated consistent identification of release points. Qualitative assessment of performance suggests this may be likened to a flicking motion of the wrist upon release. Without kinetic validation, it can be hypothesized that this motion does not maximize athlete efficiency. Whilst IMUs may have a limited capacity to quantify the release time, they can still assist in kinematic profiling, by recognizing whether inefficient release techniques are being used.

Results suggest that differences in reliability of the IMU are not associated so much with athlete equipment (wheel type or glove type) but are related to individual propulsion techniques. It is suggested that IMUs demonstrate greater agreement with the current gold standard method when used with more experienced, senior athletes, and at higher propulsion speeds. Females demonstrate different propulsion kinematics [16], which may provide the rationale as to why reliability and gender are correlated, however due to the small population sample size $(n=2)$, and the potential of systematic error, this cannot be confirmed from the results of this study. Athlete classification, which influences kinematic technique was not demonstrated to influence the reliability of the IMUs in determining contact. However, both the classifications researched had full function of their upper extremities. It is expected that level of activity limitation (and therefore classification) would be an influencing factor when concerning athletes with different types of impairment, specifically when reduced muscular function and greater physical asymmetry is present in the upper extremities.

\section{Conclusions}

Identification of key propulsion events from IMU data were found to be reliable for contact and push length, but not for release. The reliability of IMU obtained data was impacted by athlete propulsion technique. The robustness of detecting contact was not influenced by classification, wheel 
type, or glove type, however improved reliability may be associated with athlete speed, gender and variability in technique.

Acknowledgments: The authors would like to thank Keren Faulkner (Australian Paralympic Committee, Adelaide, Australia), Joseph Winter (Australian Institute of Sport, Canberra, Australia) and David Haydon (University of Adelaide, Adelaide, Australia) for their contributions to this research. The authors would also like to thank the Australian Government Training Research Program Scholarship for assisting the research.

Conflicts of Interest: The authors declare no conflict of interest.

\section{References}

1. Gutierrez, E.M.; Alm, M.; Hultling, C.; Saraste, H. Measuring seating pressure, area, and asymmetry in persons with spinal cord injury. Eur. Spine J. 2004, 13, 374-379, doi:10.1007/s00586-003-0635-7.

2. Hurd, W.J.; Morrow, M.M.; Kaufman, K.R.; An, K.N. Biomechanic evaluation of upper-extremity symmetry during manual wheelchair propulsion over varied terrain. Arch. Phys. Med. Rehabil. 2008, 89, 1996-2002, doi:10.1016/j.apmr.2008.03.020.

3. Soltau, S.L.; Slowik, J.S.; Requejo, P.S.; Mulroy, S.J.; Neptune, R.R. An investigation of bilateral symmetry during manual wheelchair propulsion. Front. Bioeng. Biotechnol. 2015, 3, 86, doi:10.3389/fbioe.2015.00086.

4. West, C.R.; Campbell, I.G.; Goosey-Tolfrey, V.L.; Mason, B.S.; Romer, L.M. Effects of abdominal binding on field-based exercise responses in Paralympic athletes with cervical spinal cord injury. J. Sci. Med. Sport 2014, 17, 351-355.

5. Van der Slikke, R.; Berger, M.; Bregman, D.; Veeger, D. Push characteristics in wheelchair court sport sprinting. Procedia Eng. 2016, 147, 730-734, doi:10.1016/j.jsams.2013.06.001.

6. Vegter, R.J.; Lamoth, C.J.; de Groot, S.; Veeger, D.H.; van der Woude, L.H. Variability in bimanual wheelchair propulsion: consistency of two instrumented wheels during handrim wheelchair propulsion on a motor driven treadmill. J. Neuroeng. Rehabil. 2013, 10, 1-12, doi: 10.1186/1743-0003-10-9.

7. Cooper, R.A. Wheelchair racing sports science: A review. JRRD 1990, 27, 295-312, doi:10.1682/JRRD.1990.07.0297.

8. Fuss, F.K. Influence of mass on the speed of wheelchair racing. Sports Eng. 2009, 12, 41-53, doi:10.1007/s12283-009-0027-2.

9. Tweedy, S. IPC Athletics Classification Project for Physical Impairments; Final Report-Stage 1; International Paralympic Committee Athletics: Bonn, Germany, 2010; p. 107.

10. Mason, B.; Lenton, J.; Leicht, C.; Goosey-Tolfrey, V. A physiological and biomechanical comparison of over-ground, treadmill and ergometer wheelchair propulsion. J. Sports Sci. 2014, 32, 78-91, doi:10.1080/02640414.2013.807350.

11. ITU World Triathlon Series. 2017 Gold Coast ITU World Paratriathlon Series. 2017. Available online: http://wts.triathlon.org/results/result/2017_gold_coast_itu_world_paratriathlon_series/310229 (accessed on 9 October 2017).

12. Wheelchair Sports NSW. 2017 Summer Down Under. 2014. Available online: http://wsnsw.org.au/2017summer-down-under/ (accessed on 9 October 2017).

13. Chow, J.W.; Millikan, T.A.; Carlton, L.G.; Morse, M.I.; Chae, W.S. Biomechanical comparison of two racing wheelchair propulsion techniques. Med. Sci. Sports Exerc. 2000, 33, 476-484, doi:10.1097/00005768200103000-00022.

14. Atkinson, G.; Nevill, A.M. Statistical methods for assessing measurement error (reliability) in variables relevant to sports medicine. Sports Med. 1998, 26, 217-238.

15. Field, A. Discovering Statistics Using SPSS; Sage Publications: Thousand Oaks, CA, USA, 2009.

16. Fay, B.T.; Boninger, M.; Cooper, R.A.; Koontz, A.M. Gender differences in the kinematic features of manual wheelchair propulsion. In Proceedings of the 24th Annual Meeting of the American Society of Biomechanics, Chicago, IL, USA, 19 July-22 July 2000.

(C) 2018 by the authors. Licensee MDPI, Basel, Switzerland. This article is an open access article distributed under the terms and conditions of the Creative Commons Attribution (CC BY) license (http://creativecommons.org/licenses/by/4.0/). 